\title{
Resident training in urology: Bipolar transurethral resection of the prostate - a safe method in learning endoscopic surgical procedure
}

\author{
Alessandro Del Rosso, Stefano Masciovecchio, Giuseppe Paradiso Galatioto, \\ Carlo Vicentini \\ University of L'Aquila, Department of Health Science, Mazzini Hospital, Urology Department, Teramo, Italy.
}

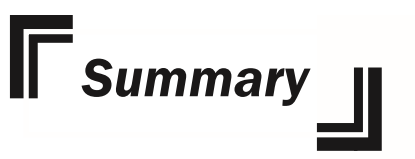

Introduction: Modern medicine uses increasingly innovative techniques that require more and more capabilities for acquisition. In the urological department is increasing the presence of patients with lower urinary tract symptoms (LUTS) and transurethral resection of the prostate (TURP) is the standard of care in their surgical treatment. We report our surgical experience and learning curve of using bipolar plasmakinetic devices in the training of urological residents to benign prostatic hyperplasia (BPH) treatment. Materials and Methods: 80 patients with benign prostatic enlargement due to $\mathrm{BPH}$ were enrolled in the study. TURP has been performed by three urological residents and by an experienced urologist. Patients were evaluated before and 6 months after the endoscopic bipolar plasmakinetic resection using the International Prostate Symptom Score (IPSS), maximum urinary flow rate $\left(Q_{\max }\right)$, postvoid residual urine (PVR) and prostate specific antigen (PSA).

Results: Overall 60 procedures were performed, 18 PlasmaKinetic (PK)-TURP procedures were completed by the three residents. In the other 42 cases the procedures were completed by the experienced urologist. In eight cases there was a capsular perforation and the experienced urologist replaced the resident to complete the resection. No complications have been reported in the procedures completed by the senior urologist. All complications caused by the residents were managed intraoperatively without changing the course of the procedure. Statistical differences were observed regarding IPSS, quality of life (QoL), and PVR at 6-month follow-up when procedures completed by urological residents were compared to those completed by the senior urologist. Conclusion: Bipolar device represents appropriate tools to acquire endoscopic skills. It is safe and it can be used at the first experience of $\mathrm{BPH}$ treatment by a resident who has not previously approached this endoscopic surgical procedure.

KEY WORDS: BPH; Resident; Surgical skills; TURP.

\section{INTRODUCTION}

Modern medicine uses increasingly innovative techniques that require more and more capabilities in order to be acquired. Consequently an objective is try to make easier the surgical technique to better manage the surgical procedure and consequently the postoperative patient's course. Technical training in any surgical procedure involves three elements: didactic learning, supervised hands-on training and unsupervised experienced.
The current organization of University departments and the new standards require that the trainees may demonstrate at least the minimum standards of competence. This would be to ensure uniform standards of training and allow comparisons among different institutions (1). In the urological departments is increasing the affluence of patients with lower urinary tract symptoms (LUTS) and benign prostatic hyperplasia $(\mathrm{BPH})$ parallel to the 
increase of the age of patients. The impact on quality of life (QoL) and health care cost justifies additional research about the use of therapeutic resources. Transurethral resection of the prostate (TURP) is the standard of care in surgical management of LUTS due to bladder outlet obstruction for prostate of 30-80 mL (2). Different type of resectoscope are actually available, roughly divided in monopolar and bipolar devices. Today is currently more widespread the use of the bipolar PlasmaKinetic (PK) TURP that appears to offer many advantages compared to the monopolar resection.

We report here our surgical experience and learning curve of using bipolar plasmakinetic devices in the training of urological residents for BPH treatment.

\section{Materials AND Methods}

\section{Patients}

From January 2009 to August 2011, a total of 80 patients with benign prostatic enlargement due to $\mathrm{BPH}$ were enrolled in the study. All patients undergo PKTURP. The study included all patients candidates for transurethral resection with a prostate volume at least of $80 \mathrm{ml}$, LUTS causing a IPSS of 15 or greater, QoL index of 2 or greater and $Q_{\max }$ less than $15 \mathrm{ml} / \mathrm{s}$. All patients were older than 50 years with symptomatic benign prostatic hyperplasia of at least 3-months duration of and who did not benefit of medical therapy with persistence or progression of urinary symptoms. Exclusion criteria included patients with urinary tract infections and patients who reported a neurogenic bladder dysfunction, abnormal digital rectal examination (DRE) findings or known prostate cancer, previous prostatic or urethral surgery or bladder stones. Written informed consent has been obtained from all subjects. Anticoagulant drugs have been discontinued at least 7 days before surgery. Patients were evaluated before and 6 months after the endoscopic resection using the IPSS bother score, the measurements of the $Q_{\max }$, PVR and PSA. We reported the experience and technical progress in treating patients with the plasmakinetic bipolar endoscopic device and evaluated the difficulties in carrying out the surgical procedures, considering patients outcome in the immediate postoperative and after six-months follow-up.

SPSS for Windows (version 10.0.7) computer package was used for statistical analysis of the data. The Wilcoxon test was used and $p<0.05$ was considered as a level of statistical significance.

\section{Surgical technique}

All the procedures were performed under spinal anesthesia, in lithotomic position and after a preoperative antibiotic prophylaxis. The endoscopic procedures were performed by three urological residents ( 20 procedures for each) and an experienced urologist (20 procedures). PK-TURP has been performed using the Gyrus Plasmakinetic Tissue Management System (Gyrus Medical Ltd., Cardiff, UK) with a $27 \mathrm{Ch}$ continuous flow resectoscope and a U-shaped cutting loop. Once connected, the generator was automatically set to $160 \mathrm{~W}$ for cutting and $80 \mathrm{~W}$ for coagulation. Saline solution was used for irri- gation. After the introduction of the resectoscope, the surgeon always provided to mark the ureteral orifices. The incision at 6 o'clock position was the first step of the endoscopic procedure with a cut extended from bladder neck to the veru montanum, deep to the surgical capsule. Thereafter the incision at the 12 o'clock position was performed, involving a $180^{\circ}$ rotation of the sheath being careful to the adequate depth and length of the incision. Finally we proceeded to resection of the prostatic lateral lobes. At the end of the procedure a $20 \mathrm{Ch}$ 3 -way catheter was inserted. Postoperatively a continuous saline bladder irrigation was used until the efflux was sufficiently clear, with subsequent catheter removal and patient discharge when urine were clear.

\section{Residents training}

Prior to perform endoscopic procedures in the operating room, the urological residents were given didactic lessons on the surgical technique at an academic teaching hospital (3). Following these lessons, the residents practiced the surgical device and, once the attending surgeon determined that the residents were able to perform the procedure safely, they performed a surgical endoscopic resection on the patient in the operating room, always with the senior urologist supervision.

\section{RESULTS}

Baseline and immediate postoperative parameters are reported in Table 1. All patients completed the sixmonths follow-up. Overall 80 procedures performed, the three residents completed a mean of 6 PK-TURP for each one, without any intervention of the experienced urologist, but only with his supervision. In the first 14 cases for each resident, the procedures were completed by the experienced urologist due to different reasons (loss of orientation, low speed of resection, bleeding, capsular perforation). Out of all the endoscopic PKTURP treatments, a capsular perforation was observed in

Table 1.

Baseline and immediate postoperative parameters of the patients.

\begin{tabular}{|l|c|c|}
\hline Age (years) & Mean & Range \\
\hline Total prostate volume $(\mathrm{g})$ & 66.3 & $51-78$ \\
\hline Transitional zone volume $(\mathrm{g})$ & 51.6 & $32-68$ \\
\hline Resection time $(\mathrm{min})$ & 28.5 & $18-44$ \\
\hline Resection weight $(\mathrm{g})$ & 55 & $26-105$ \\
\hline Preoperative PSA $(\mathrm{ng} / \mathrm{ml})$ & 31 & $22-40$ \\
\hline Preoperative mean $\mathbf{Q}_{\max }(\mathrm{ml} / \mathrm{s})$ & 3.45 & $0.8-6.3$ \\
\hline Preoperative mean $\mathbf{P V R}(\mathrm{ml})$ & 7.2 & $2.6-12.4$ \\
\hline Catheterization time $(\mathrm{day})$ & 160 & $50-280$ \\
\hline Hospital stay $($ day) & 2.4 & $2-4$ \\
\hline Hemoglobin decrease $(\mathrm{g} / \mathrm{dl})$ & 3.2 & $3-5$ \\
\hline Blood transfusion & 1.7 & $0.7-2.7$ \\
\hline TUR syndrome & None & - \\
\hline
\end{tabular}


Table 2.

Perioperative, immediate complications and postoperative characteristics at 6-months follow-up of the different type of procedures and surgeon.

\begin{tabular}{|l|c|c|c|}
\hline & $\begin{array}{c}\text { Procedures not completed } \\
\text { by residents alone } \\
\text { Group 1 }\end{array}$ & $\begin{array}{c}\text { Procedures completed } \\
\text { by residents alone (with supervision) } \\
\text { Group 2 }\end{array}$ & $\begin{array}{c}\text { Procedures completed } \\
\text { by experienced urologist } \\
\text { Group 3 }\end{array}$ \\
\hline No. of procedures & $42 / 60(70 \%)$ & $18 / 60(30 \%)$ & $20 / 20(100 \%)$ \\
\hline Capsular perforation & $3 / 60(5 \%)$ & $5 / 60(8.3 \%)$ & $0 / 20(-)$ \\
\hline Mean $\mathbf{Q}_{\mathbf{m a x}}, \mathbf{m l} / \mathbf{s}^{*}$ & $20.1(15.3-26.2)$ & $22.7(17.4-32.6)$ & $25.6(19.5-34.2)$ \\
\hline Mean IPSS & $2.8(1-3)$ & $3.1(2-3)$ & $2.8(2-3)$ \\
\hline Mean QoL & $2.3(1-3)$ & $2.4(1-3)$ & $2.2(0-3)$ \\
\hline Mean PVR, $\mathbf{m l}$ & $15(0-40)$ & $20(0-50)$ & $10(0-30)$ \\
\hline Mean PSA, ng/ml & $1.5(0.4-2.3)$ & $1.5(0.9-2.1)$ & $1.2(0.6-1.7)$ \\
\hline Mean resected weight $(\mathrm{g})^{* *}$ & $32(27-38)$ & $27(22-36)$ & $42(28-48)$ \\
\hline
\end{tabular}

$* p<0.05$ group 1 vs group $3 ; * * p<0.05$ group 2 vs group 3.

eight cases. In these cases, the experienced urologist replaced the resident in order to complete the resection (Table 2). All the complications caused by the residents were managed intraoperatively without changing the course of the procedure. Comparing endourological procedures carried out by the resident alone with those performed by the senior urologist, the latter showed no problems or complications of the resection. Mean catheterization time was 2.4 days in the PK-TURP and mean hospital stay was 3.2 days. No cases of TUR syndrome have been observed.

Considering the immediate postoperative and 6 months follow-up, in all cases there was a normal course of the patient both in the time of catheterization that in hospitalization. After 6 months, comparing the three groups: 1) procedures not completed by the resident, 2) procedures completed alone with supervision and 3) procedures completed by the experienced urologist, $Q_{\text {max }}$ value was significantly different between group 1 and 3 $(\mathrm{p}<0.05)$, no significant differences have been reported between the other two groups. No differences were observed regarding IPSS, QoL, PVR and preoperative and postoperative 6-months PSA. Moreover, we report significant differences $(\mathrm{p}<0.05)$ in the resected weight between group 2 and group 3 .

\section{Discussion}

Endourology is one of the most difficult techniques to learn. Safe and effective performance of diagnostic and therapeutic endourological procedures requires longterm practical experience.

Training opportunities for residents and urologists are the way to increase their experience in the surgical approach (4). As just reported, the technical training in any procedure involves three elements: 1) didactic learning, 2) supervised hands-on training and 3) unsupervised experience. Obviously the third parameter continues throughout the individual professional career, and so, the first two elements are basic for proper growth of a resident. Nowadays, the standardization of education is increasingly important for the education of all professionals (1). Although didactic training methods can accelerate the evolution of procedural skills, they cannot substitute the other elements, considering the hands-on experience through which technical skills, including three-dimensional awareness and hand-eye coordination, develop $(5,6)$. Currently, the most frequently used models are the synthetic organ models for ureterorenoscopy and transurethral resection of the prostate. However the use of these tools has a limited value due to the lack of bleeding and the unrealistic force feedback. Furthermore local and national regulation in relation to the use of animal organs in the hospital and use of instruments in animal urinary tracts must be also considered. As a consequence the main way to gain experience remains the real contact with the patient and the surgical treatment of his pathology (7).

Currently BPH is one of the most common disease that affect men beyond middle age and TURP is the standard of care in the surgical management of LUTS due to bladder outlet obstruction. Regarding this endoscopic procedure, significant technical improvements during the past 15 years have been introduced and the bipolar technology has become a safe and effective procedure in alternative to conventional monopolar TURP (8).

Our training course allowed us to learn and approach the endoscopic surgical technique with the new endoscopic modern devices. Our data showed that there is a greater ease of training with the bipolar instrument, also confirmed by the increasing number of resections that have been completed by the residents alone with onlly supervision of an expert. In addition, only minor complications occurred during the endoscopic procedures confirming the easier handling for the PK device. In fact the learning curve seems to confirm that already an approximate number of about 20 procedures resulted in a good capacity of surgical management. In fact the latest procedures of this series were substantially completed by the residents who could complete them autonomously, although under the supervision of an experienced urologist. A six-months follow-up, although still limited, seems adequate to confirm the success of the procedure even in cases in which the resident has completed the 
resection alone by himself, without needing any direct aid for proper management of the endoscopic procedure. Clearly, results cannot be comparable to the surgical management obtained by an experienced urologist, because significant differences in terms of mean $\mathrm{Q}_{\max }$ and resected weight were observed between the procedures entirely performed by residents and those performed by an experienced urologist. These results suggests that the bipolar resector seems to be a good tool for training because it is easy to handle and speeds the learning process.

\section{Conclusions}

In our experience, bipolar devices represent a very important and appropriate way to acquire the endoscopic skills. It is possible to use this endoscopic device by residents even at their first experience of BPH treatment.

\section{REFERENCES}

1. Le CQ, Lightner DJ, VanderLei L, et al. The current Role of medical simulation in American Urological Residency Training programs: an assessment by program directors. J Urol. 2007; 177:288-91.
2. Yu X, Elliott SP, Wilt TJ, et al. Practice patterns in benign prostatic hyperplasia surgical therapy: the dramatic increase in minimally invasive technologies. J Urol. 2008; 180:241-5.

3. Alley JR, Stucky CC, Moncure M. Teaching Surgical Residents Dome-Down Laparoscopic Cholecystectomy in an Academic Medical Center. J Soc Laparoendoscopic Surg. 2008; 12:368-371.

4. Schreuder HW, Oei G, Maas M, et al. Implementation of simulation in surgical practice: Minimally invasive surgery has taken the lead: the Dutch experience. Med Teacher. 2011; 33:105-15.

5. El-Hakim A, Elhilali MM. Holmium laser enucleation of the prostate can be taught: the first learning experience. BJU International. 2002; 90:863-869.

6. Kössi J, Luostarinen M. Virtual reality laparoscopic simulator as an aid in surgical resident education: two years' experience. Scand J Surg. 2009; 98:48-54.

7. Rodríguez-Sanjuán JC, Palazuelos CM, Fernández-Díez MJ. et al. Assessment of resident training in laparoscopic surgery based on a digestive system anastomosis model in the laboratory. CIR ESP. 2010; $87: 20-25$

8. Rassweiller J, Schlze M, Stock C, et al. Bipolar transurethral resection of the prostate-technical modifications and early clinical experience. Minim Invasive Ther Allied Technol. 2007; 16:11-21.

\section{Correspondence}

Alessandro Del Rosso, MD (Corresponding Author)

delrossoa@tiscali.it

Stefano Masciovecchio, MD

Giuseppe Paradiso Galatioto, MD

Carlo Vicentini, MD

Mazzini Hospital, Department of Urology, Italy Square, Teramo, Italy 